\title{
ABSOLUTE SUMMABILITY OF SOME SERIES RELATED TO A FOURIER SERIES
}

\author{
H. P. DIKSHIT
}

(Received 10 October 1969)

Communicated by B. Mond

\section{Definitions and notations}

Let $\left\{p_{n}\right\}$ be a given sequence of constants, real or complex, such that $P_{n}=p_{0}+p_{1}+\cdots+p_{n} \neq 0, P_{-1}=p_{-1}=0$, then

$$
t_{n}=\sum_{k=0}^{n} P_{k} a_{n-k} / P_{n}
$$

defines the sequence $\left\{t_{n}\right\}$ of $\left(N, p_{n}\right)$ means of $\sum_{n} a_{n}$. The series $\sum_{n} a_{n}$ is said to be summable $\left|N, p_{n}\right|$, if $\left\{t_{n}\right\} \in B V$, i.e., $\sum_{n}\left|t_{n}-t_{n-1}\right|<\infty$.

In the special case in which

$$
p_{n}=\left(\begin{array}{c}
n+\delta-1 \\
\delta-1
\end{array}\right)=\frac{\Gamma(n+\delta)}{\Gamma(n+1) \Gamma(\delta)} ; \delta>-1,
$$

the $\left(N, p_{n}\right)$ mean reduces to the familiar $(C, \delta)$ mean. Thus $\left|N, p_{n}\right|$ summability is the same as $|C, \delta|$ summability, if $\left\{p_{n}\right\}$ is defined by (1.2).

Let $f(t)$ be a periodic function with period $2 \pi$, integrable $(L)$ over $(-\pi, \pi)$ and

Then the allied series is

$$
f(t) \sim \frac{1}{2} a_{0}+\sum_{n=1}^{\infty}\left(a_{n} \cos n t+b_{n} \sin n t\right)=\sum_{n=0}^{\infty} A_{n}(t) .
$$

$$
\sum_{n=1}^{\infty}\left(b_{n} \cos n t-a_{n} \sin n t\right)=\sum_{n=1}^{\infty} B_{n}(t) .
$$

We use the following notations:

$$
\begin{aligned}
\phi(t) & =\frac{1}{2}\{f(x+t)+f(x-t)\}, \phi^{*}(t)=\phi(t)-\phi(+0) ; \\
\psi(t) & =\frac{1}{2}\{f(x+t)-f(x-t)\} ; P_{n}^{*}=\sum_{k=0}^{n}\left|p_{k}\right| ; \\
S_{n} & =\frac{1}{P_{n}} \sum_{k=0}^{n} \frac{P_{k}}{k+1} ; S_{n}^{*}=\frac{1}{\left|P_{n}\right|} \sum_{k=0}^{n} \frac{\left|P_{k}\right|}{k+1} .
\end{aligned}
$$


If $P_{n}^{*}=O\left(\left|P_{n}\right|\right),\left\{R_{n}\right\} \equiv\left\{(n+1) p_{n} / P_{n}\right\} \in B V$ and for some real $\delta$,

$$
\left|P_{k}\right| \sum_{n=k}^{\infty} \frac{1}{n^{1-\delta}\left|P_{n}\right|} \leqq K k^{\delta}, k=1,2, \cdots ;
$$

then we write $\left\{p_{n}\right\} \in \mathscr{C}^{\delta}$.

We put $\lambda_{n}^{\delta}(t)=n^{\delta} \sin n t ; \tilde{\lambda}_{n}^{\delta}(t)=n^{\delta} \cos n t ; \tau=[\pi / t]$, the greatest integer not greater than $\pi / t$.

By ' $F(t) \in B V(a, b)$ ', we mean that $F(t)$ is a function of bounded variation in $(a, b)$ and ' $\left\{\mu_{n}\right\} \in B$ ' means that $\left\{\mu_{n}\right\}$ is a bounded sequence. $\Delta \mu_{n}=\mu_{n}-\mu_{n+1}$.

$K$ denotes a positive constant, not necessarily the same at each occurrence.

\section{Introduction}

Concerning the $|C|$-summability of $\sum_{n} n^{\alpha} A_{n}(t)$ and $\sum_{n} n^{\alpha} B_{n}(t)$, we have the following results due to Mohanty [5]. ${ }^{1}$

THEOREM A. If

$$
0<\alpha<1 \text { and } \int_{0}^{\pi} t^{-\alpha}|d \phi(t)| \leqq K,
$$

then $\sum_{n} n^{x} A_{n}(x)$ is summable $|C, \beta|$ for every $\beta>\alpha$.

THEOREM B. If

$$
0<\alpha<1, \psi(+0)=0 \text { and } \int_{0}^{\pi} t^{-\alpha}|d \psi(t)| \leqq K,
$$

then $\sum_{n} n^{a} B_{n}(x)$ is summable $|C, \beta|$ for every $\beta>\alpha$.

The case $\alpha=0$ of Theorem A corresponds to an earlier result of Bosanquet [1], which follows as a special case of the following.

Theorem C. If $\phi(t) \in B V(0, \pi)$ and $\left\{p_{n}\right\} \in \mathscr{C}^{0}$, then $\sum_{n} A_{n}(x)$ is summable $\left|N, p_{n}\right|$.

As pointed out in section 7 of the present paper, Theorem $C$ is obtained by a slight modification in the proof of one of our main results given in this paper. Incidently, this provides a much shorter proof of a result due to Si-Lei ([9], Theorem 1), which is a generalisation of some of the earlier results due to Pati [6], [7], Varshney [10] and Dikshit [2], when we demonstrate in section 7 that the hypotheses used by Si-Lei imply that $\left\{p_{n}\right\} \in \mathscr{C}^{0}$.

In view of Theorem $C$ and the corresponding result for $|C|$-summability due to Bosanquet [1], it is natural to expect from Theorem $\mathrm{A}$ and Theorem $\mathrm{B}$ that the hypotheses (2.1) and (2.2) may lead to $\left|N, p_{n}\right|$ summability of $\sum_{n} n^{\alpha} A_{n}(t)$ and

1 We write $\int_{0}^{\pi}$ for $\lim _{\varepsilon \rightarrow+0} \int_{\varepsilon}^{\pi}$. 
$\sum_{n} n^{\alpha} B_{n}(t)$, respectively, if $\left\{p_{n}\right\} \in \mathscr{C}^{\alpha}$ and that such results may include as a special case Theorem A or Theorem B. The object of the present paper is to show that this is indeed true. That Theorem A and Theorem B are special cases of our Theorem 1 and Theorem 2, respectively follows when we observe that

$$
\left\{p_{n}\right\} \equiv\left\{\left(\begin{array}{c}
n+\beta-1 \\
\beta-1
\end{array}\right)\right\} \in \mathscr{C}^{\alpha}, \beta>\alpha>0,
$$

and $\left|N, p_{n}\right|$ for such a $\left\{p_{n}\right\}$ is the same as $|C, \beta|$.

\section{The main results}

We prove the following.

THEOREM 1. If (2.1) holds and $\left\{p_{n}\right\} \in \mathscr{C}^{\alpha}$ then $\sum_{n} n^{\alpha} A_{n}(x)$ is summable $\left|N, p_{n}\right|$.

THeOrem 2. If (2.2) holds and $\left\{p_{n}\right\} \in \mathscr{C}^{\alpha}$ then $\sum_{n} n^{\alpha} B_{n}(x)$ is summable $\left|N, p_{n}\right|$.

\section{Lemmas}

LEMMA 1. If $0<m \leqq n$, and $0<\alpha<1$, then uniformly in $0<t \leqq \pi$,

$$
\left|\sum_{k=m}^{n} k^{\alpha-1} \exp (i k t)\right| \leqq K t^{-\alpha}
$$

Proof. The lemma follows, when we observe that

$$
\begin{gathered}
\left|\sum_{k=m}^{n} k^{\alpha-1} \exp (i k t)\right| \leqq \sum_{k=m}^{\tau} k^{\alpha-1}+K \tau^{\alpha-1} \max _{\tau<v \leqq n}\left|\sum_{k=\tau+1}^{v} \exp (i k t)\right| \\
\leqq K \tau^{\alpha} .
\end{gathered}
$$

LEMMA 2. If $P_{n}^{*}=O\left(\left|P_{n}\right|\right)$, then uniformly in $0<t \leqq \pi$,

$$
\left|\sum_{k=0}^{v} P_{k}(n-k)^{\alpha-1} \exp i(n-k) t\right| \leqq K t^{-\alpha}\left|P_{v}\right|
$$

where $0 \leqq v<n$ and $0<\alpha<1$.

Proof. We have by Abel's transformation and Lemma 1,

$$
\begin{aligned}
\left|\sum_{k=0}^{v} P_{k}(n-k)^{\alpha-1} \exp i(n-k) t\right| \\
\quad \leqq \sum_{k=0}^{v-1}\left|p_{k+1} \| \sum_{\mu=0}^{k}(n-\mu)^{\alpha-1} \exp i(n-\mu) t\right|+\left|P_{v}\right|\left|\sum_{\mu=0}^{v}(n-\mu)^{\alpha-1} \exp i(n-\mu) t\right| \\
\quad \leqq K t^{-\alpha} P_{v}^{*} .
\end{aligned}
$$

The lemma now follows when we appeal to the hypothesis: $P_{n}^{*}=O\left(\left|P_{n}\right|\right)$. 
LeMma 3. If $\left\{p_{n}\right\}$ is any sequence such that $P_{n}^{*}=O\left(\left|P_{n}\right|\right)$, then uniformly in $0<t \leqq \pi$

$$
\left|\sum_{k=0}^{v} P_{k} \exp i(n-k) t\right| \leqq K t^{-1}\left|P_{v}\right|
$$

where $0 \leqq v$ and $n$ is any integer.

The proof of Lemma 3 is similar to that of Lemma 2.

LEMMA 4. For any sequence $\left\{p_{n}\right\}$ such that $P_{n}^{*}=O\left(\left|P_{n}\right|\right)$, the statement $\left\{S_{n}\right\} \in B V$ implies $\left\{S_{n}^{*}\right\} \in B$.

Lemma 4 is the same as Lemma 2 in [3].

LemmA 5. If $\propto>0, \eta>0$, then necessary and sufficient conditions that

$$
\int_{0}^{\eta} t^{-\alpha}|d \psi(t)| \leqq K \text { and } \psi(+0)=0,
$$

are that (i) $t^{-\alpha} \psi(t) \in B V(0, \eta),{ }^{2}$ and (ii) $t^{-\alpha-1}|\psi(t)|$ should be integrable in $(0, \eta)$. Lemma 5 is given in [5].

\section{Proof of Theorem 1}

We have

$$
\begin{aligned}
t_{n}-t_{n-1} & =\sum_{k=0}^{n-1}\left(\frac{P_{k}}{P_{n}}-\frac{P_{k-1}}{P_{n-1}}\right) a_{n-k} \\
& =\frac{1}{P_{n} P_{n-1}} \sum_{k=0}^{n-1}\left(P_{n} p_{k}-p_{n} P_{k}\right) a_{n-k} .
\end{aligned}
$$

Integrating by parts, we have

$$
\begin{aligned}
n^{\alpha} A_{n}(x) & =\frac{2}{\pi} \int_{0}^{\pi} \phi(t) n^{\alpha} \cos n t d t \\
& =-\frac{2}{\pi} \int_{0}^{\pi} n^{\alpha-1} \sin n t d \phi(t) .
\end{aligned}
$$

Thus for the series $\sum_{n} n^{x} A_{n}(x)$,

$$
\begin{aligned}
\sum_{n}\left|t_{n}-t_{n-1}\right| & =\frac{2}{\pi} \sum_{n}\left|\int_{0}^{\pi}\left\{\frac{1}{P_{n} P_{n-1}} \sum_{k=0}^{n-1}\left(P_{n} p_{k}-p_{n} P_{k}\right) \lambda_{n-k}^{\alpha-1}(t)\right\} d \phi(t)\right| \\
& \leqq \int_{0}^{\pi} \sum_{n}\left|\frac{1}{P_{n} P_{n-1}} \sum_{k=0}^{n-1}\left(P_{n} p_{k}-p_{n} P_{k}\right) \lambda_{n-k}^{\alpha-1}(t)\right||d \phi(t)| .
\end{aligned}
$$

Since $\int_{0}^{\pi} t^{-\alpha}|d \phi(t)| \leqq K$, in order to prove Theorem 1 it is sufficient to show that uniformly in $0<t \leqq \pi$

2 That is, in the interval $0<t \leqq \eta$. 


$$
\sum \equiv t^{\alpha} \sum_{n}\left|\frac{1}{P_{n} P_{n-1}} \sum_{k=0}^{n-1}\left(P_{n} p_{k}-p_{n} P_{k}\right) \lambda_{n-k}^{\alpha-1}(t)\right| \leqq K .
$$

Now (cf. [2], p. 168)

$$
\begin{aligned}
\Sigma \leqq & t^{\alpha} \sum_{n=1}^{\infty} \frac{1}{(n+1)\left|P_{n-1}\right|}\left|\sum_{k=0}^{n-1} P_{k}\left(R_{k}-R_{n}\right) \lambda_{n-k}^{\alpha-1}(t)\right| \\
& +t^{\alpha} \sum_{n=1}^{\tau} \frac{1}{(n+1)\left|P_{n-1}\right|}\left|\sum_{k=0}^{n-1} p_{k} \lambda_{n-k}^{\alpha}(t)\right| \\
& +t^{\alpha} \sum_{n=\tau+1}^{\infty} \frac{1}{(n+1)\left|P_{n-1}\right|}\left|\sum_{k=0}^{\tau-1} p_{k} \lambda_{n-k}^{\alpha}(t)\right| \\
& +t^{\alpha} \sum_{n=\tau+1}^{\infty} \frac{1}{(n+1)\left|P_{n-1}\right|}\left|\sum_{k=\tau}^{n} p_{k} \lambda_{n-k}^{\alpha}(t)\right| \\
= & \Sigma_{1}+\Sigma_{2}+\Sigma_{3}+\Sigma_{4},
\end{aligned}
$$

say. By a change of order of summation, we have

$$
\begin{aligned}
\Sigma_{1} & =t^{\alpha} \sum_{n=1}^{\infty} \frac{1}{(n+1)\left|P_{n-1}\right|}\left|\sum_{v=0}^{n-1} \Delta R_{v} \sum_{k=0}^{v} P_{k} \lambda_{n-k}^{\alpha-1}(t)\right| \\
& \leqq K \sum_{n=1}^{\infty} \frac{1}{(n+1)\left|P_{n-1}\right|} \sum_{v=0}^{n-1}\left|\Delta R_{v}\right|\left|P_{v}\right| \\
& =K \sum_{v=0}^{\infty}\left|\Delta R_{v}\right|\left|P_{v}\right| \sum_{n=v+1}^{\infty} \frac{1}{(n+1)\left|P_{n-1}\right|} \\
& \leqq K \sum_{v=0}^{\infty}\left|\Delta R_{v}\right| \leqq K,
\end{aligned}
$$

by virtue of Lemma 2 and the hypothesis that $\left\{p_{n}\right\} \in \mathscr{C}^{x}$.

Since $|\sin (n-k) t| \leqq n t$ for relevant $k$, we have

$$
\Sigma_{2} \leqq t^{\alpha+1} \sum_{n=1}^{\tau} \frac{n^{\alpha+1}}{n\left|P_{n-1}\right|} P_{n-1}^{*} \leqq K,
$$

by virtue of the hypothesis that $\left\{p_{n}\right\} \in \mathscr{C}^{x}$.

Next, we observe that

$$
\Sigma_{3} \leqq K t^{\alpha} P_{\tau}^{*} \sum_{n=\tau+1}^{\infty} \frac{1}{n^{\alpha-1}\left|P_{n-1}\right|} \leqq K
$$

by virtue of the hypothesis that $\left\{p_{n}\right\} \in \mathscr{C}^{\alpha}$.

Since $p_{k}=(k+1)^{-1} R_{k} P_{k}$, we have by Abel's transformation 


$$
\begin{aligned}
\left|\sum_{k=\tau}^{n} p_{k} \lambda_{n-k}^{\alpha}(t)\right| & \\
= & \left|\sum_{k=\tau}^{n-1} \Delta\left(\frac{R_{k}}{k+1}\right) \sum_{v=\tau}^{k} P_{v} \lambda_{n-v}^{\alpha}(t)+\frac{R_{n}}{n+1} \sum_{v=\tau}^{n-1} P_{v} \lambda_{n-v}^{\alpha}(t)\right| \\
& \leqq K n^{\alpha} t^{-1} \sum_{k=\tau}^{n-1}\left\{\frac{\left|\Delta R_{k}\right|}{k+1}+\frac{\left|R_{k+1}\right|}{(k+1)(k+2)}\right\}\left|P_{k}\right|+K n^{\alpha-1} t^{-1}\left|P_{n-1}\right|
\end{aligned}
$$

by Abel's Lemma, Lemma 3 and the hypothesis that $\left\{p_{n}\right\} \in \mathscr{C}^{x}$.

Thus, finally

$$
\begin{aligned}
\Sigma_{4} \leqq & K t^{\alpha-1} \sum_{n=\tau+1}^{\infty} \frac{1}{n^{1-\alpha}\left|P_{n-1}\right|} \sum_{k=\tau}^{n} \frac{\left|P_{k}\right|}{k}\left\{\left|\Delta R_{k}\right|+\frac{1}{k}\right\} \\
& +K t^{\alpha-1} \sum_{n=\tau+1}^{\infty} \frac{1}{n^{2-\alpha}} \\
\leqq & K t^{\alpha-1} \sum_{k=\tau}^{\infty}\left\{\left|\Delta R_{k}\right|+\frac{1}{k}\right\} \frac{\left|P_{k}\right|}{k} \sum_{n=k+1}^{\infty} \frac{1}{n^{1-\alpha}\left|P_{n-1}\right|}+K \\
\leqq & K t^{\alpha-1} \sum_{k=\tau}^{\infty}\left\{\left|\Delta R_{k}\right|+\frac{1}{k}\right\} k^{\alpha-1}+K \\
\leqq & K \sum_{k=\tau}^{\infty}\left|\Delta R_{k}\right|+K \leqq K,
\end{aligned}
$$

by virtue of the hypothesis that $\left\{p_{n}\right\} \in \mathscr{C}^{x}$.

Combining $(5.2)-(5.6)$, we prove $(5.1)$, which completes the proof of Theorem 1 .

\section{Proof of Theorem 2}

Integrating by parts and observing that $\psi(+0)=0$, we have [5]

$$
\begin{aligned}
n^{\alpha} B_{n}(x) & =\frac{2}{\pi} \int_{0}^{\pi} \psi(t) n^{\alpha} \sin n t d t \\
& =-\frac{2}{\pi} \psi(\pi) n^{\alpha-1} \cos n \pi+\frac{2}{\pi} \int_{0}^{\pi} n^{\alpha-1} \cos n t d \psi(t) .
\end{aligned}
$$

As in the proof of Theorem 1 , for the series $\sum_{n} n^{x} B_{n}(x)$, we have

$$
\begin{aligned}
\sum_{n}\left|t_{n}-t_{n-1}\right| \leqq & |\psi(\pi)| \sum_{n}\left|\frac{1}{P_{n} P_{n-1}} \sum_{k=0}^{n-1}\left(P_{n} p_{k}-p_{n} P_{k}\right) \tilde{\lambda}_{n-k}^{\alpha-1}(\pi)\right| \\
& +\int_{0}^{\pi} \sum_{n}\left|\frac{1}{P_{n} P_{n-1}} \sum_{k=0}^{n-1}\left(P_{n} p_{k}-p_{n} P_{k}\right) \tilde{\lambda}_{n-k}^{\alpha-1}(t)\right||d \psi(t)| .
\end{aligned}
$$


Since $\int_{0}^{\pi} t^{-x}|d \psi(t)| \leqq K$, in order to prove Theorem 2 , it is sufficient to show that uniformly in $0<t \leqq \pi$

$$
\sum_{n}\left|\frac{1}{P_{n} P_{n-1}} \sum_{k=0}^{n-1}\left(P_{n} p_{k}-p_{n} P_{k}\right) \tilde{\lambda}_{n-k}^{\alpha-1}(t)\right| \leqq K .
$$

The proof of (6.1) follows from the preceeding section, when one observes that the proof of (5.1) with a slight modification remains valid even if $\tilde{\lambda}_{n-k}^{\alpha-1}(t)$ is replaced by $\tilde{\lambda}_{n-k}^{\alpha-1}(t)$.

This completes the proof of Theorem 2 .

7.

In view of Lemma 5, our Theorem 1 and Theorem 2 are equivalent to the following, respectively (cf. Theorem $1 \mathrm{a}$ and Theorem 2a due to Mohanty [5] and Theorem IV and Theorem III due to Salem and Zygmund [8]).

THEOREM 1'. If $\left\{p_{n}\right\} \in \mathscr{C}^{\alpha}$ and

$$
0<\alpha<1, t^{-\alpha} \phi^{*}(t) \in B V(0, \pi) \text { and } \int_{0}^{\pi} t^{-\alpha-1}\left|\phi^{*}(t)\right| d t \leqq K,
$$

then $\sum_{n} n^{\alpha} A_{n}(x)$ is summable $\left|N, p_{n}\right|$.

THEOREM 2'. If $\left\{p_{n}\right\} \in \mathscr{C}^{\alpha}$ and

$$
0<\alpha<1, t^{-\alpha} \psi(t) \in B V(0, \pi) \text { and } \int_{0}^{\pi} t^{-\alpha-1}|\psi(t)| d t \leqq K,
$$

then $\sum_{n} n^{\alpha} B_{n}(x)$ is summable $\left|N, p_{n}\right|$.

Under a condition similar to the last condition of $(2.1)^{\prime}$ with $\alpha=0$, recently the present author has deduced from the proof given in [2], a result concerning $\left|N, p_{n}\right|$ summability of a series associated with $\sum_{n} A_{n}(t)$ in [4].

It follows from the proof of Theorem 1 that in order to prove Theorem $\mathrm{C}$, it is sufficient to prove (5.1) uniformly in $0<t \leqq \pi$, when $\alpha=0$. Using the technique of proof of Lemma 2, we observe that if $\left\{p_{n}\right\} \in \mathscr{C}^{x}$ and $0 \leqq v<n$, then

$$
\left|\sum_{k=0}^{v} P_{k} \lambda_{n-k}^{-1}(t)\right| \leqq K\left|P_{v}\right|
$$

since $\sum_{k=a}^{b}\left|\lambda_{k}^{-1}(t)\right| \leqq K$ for any $b \geqq a>0$. Therefore $\sum_{1} \leqq K$ in the case $\alpha=0$ also. The proofs of $\sum_{2} \leqq K, \sum_{3} \leqq K$ and $\sum_{4} \leqq K$, when $\alpha=0$, run exactly parallel to those given in (5.4) $-(5.6)$.

This completes the proof of Theorem C.

Finally, to demonstrate that the hypotheses used by Si-Lei for the proof of his Theorem 1 in [9], imply that $\left\{p_{n}\right\} \in \mathscr{C}^{\alpha}$, we have the following.

If $\left\{p_{n}\right\}$ is any sequence such that $P_{n}^{*}=O\left(\left|P_{n}\right|\right)$, then $\left\{S_{n}\right\} \in B V$ implies that

$$
\left|P_{k}\right| \sum_{n=k+1}^{\infty} \frac{1}{n\left|P_{n-1}\right|} \leqq K, \quad k=0,1,2, \cdots
$$


Since $P_{n}^{*}=O\left(\left|P_{n}\right|\right)$,

$$
\begin{aligned}
\left|P_{k}\right| \sum_{n=k+1}^{M} \frac{1}{n\left|P_{n-1}\right|} \leqq & K\left|P_{k}\right| \sum_{n=k+1}^{M} \frac{\left|P_{n-1}\right|}{n}\left(P_{n-1}^{*}\right)^{-2} \\
\leqq & K\left|P_{k}\right| \sum_{n=k+1}^{M-1}\left\{\left(P_{n-1}^{*}\right)^{-2}-\left(P_{n}^{*}\right)^{-2}\right\} \sum_{v=1}^{n} \frac{\left|P_{v-1}\right|}{v} \\
& +\frac{K}{P_{k}^{*}} \sum_{v=1}^{k+1} \frac{\left|P_{v-1}\right|}{v}+K \frac{\left|P_{k}\right|}{\left(P_{M-1}^{*}\right)^{2}} \sum_{v=1}^{M} \frac{\left|P_{v-1}\right|}{v} \\
\leqq & K\left|P_{k}\right| \sum_{n=k+1}^{M} \frac{\left|p_{n}\right|}{P_{n}^{*} P_{n-1}^{*}} S_{n-1}^{*}+K S_{k}^{*}+K S_{M-1}^{*} \\
\leqq & K\left|P_{k}\right| \sum_{n=k+1}^{M}\left(\frac{1}{P_{n-1}^{*}}-\frac{1}{P_{n}^{*}}\right)+K \leqq K, \quad M \rightarrow \infty
\end{aligned}
$$

since by Lemma $4,\left\{S_{n}^{*}\right\} \in B$.

\section{References}

[1] L. S. Bosanquet, 'Note on the absolute summability ( $C$ ) of a Fourier series', J. London Math. Soc. 11 (1936), 11-15.

[2] H. P. Dikshit, 'On the absolute summability of a Fourier series by Nörlund means', Math. Z. 102 (1967), 166-170.

[3] H. P. Dikshit, 'On the absolute Nörlund summability of a Fourier series and its conjugate series', Kódai Math. Sem. Rep. 20 (1968), 448-453.

[4] H. P. Dikshit, 'Absolute summability of a series associated with a Fourier series', Proc. Amer. Math. Soc. 22 (1969), 316-318.

[5] R. Mohanty, 'The absolute Cesàro summability of some series associated with a Fourier series and its allied series', J. London Math. Soc. 25 (1950), 63-67.

[6] T. Pati, 'On the absolute Nörlund summability of a Fourier series', J. London Math. Soc. 34 (1959), 153-160; Addendum, 37 (1962), 256.

[7] T. Pati, 'On the absolute summability of a Fourier series by Nörlund means', Math. $Z$. 88 (1965), 244-249.

[8] R. Salem and A. Zygmund, 'Capacity of sets and Fourier series', Trans. Amer. Math. Soc. 59 (1946), 23-41.

[9] W. Si-Lei (Szu-Lei), 'On the absolute Nörlund summability of a Fourier series and its conjugate series', Acta Math. Sinica, 15 (1965), 559-573.

[10] O. P. Varshney, 'On the absolute Nörlund summability of a Fourier series', Math. Z. 83 (1964), 18-24.

University of Allahabad, Allahabad (India) and

University of Jabalpur, Jabalpur (India) 